\title{
Efficacy and pharmacokinetic activity of frovatriptan compared to rizatriptan in patients with moderate-to-severe migraine
}

This article was published in the following Dove Press journal:

Drug Design, Development and Therapy

21 July 2014

Number of times this article has been viewed

Lidia Savi ${ }^{\prime}$

Selene Mogavero ${ }^{2}$

Colin Gerard Egan²

'Department of Neuroscience, University of Turin, Turin, Italy;

${ }^{2}$ Primula Multimedia SRL, Pisa, Italy
Correspondence: Colin Gerard Egan

Primula Multimedia SRL, 22/B Via

Giuseppe Ravizza, Ospedaletto,

Pisa 56I2I, Italy

Tel +39050965 6242

Fax +39050316 3810

Email cegan@primulaedizioni.it
Background: Migraine is a painful neurological disorder that affects over $10 \%$ of the general population. Frovatriptan and rizatriptan are antimigraine agents belonging to the triptan class. Although previous studies have independently compared the efficacy of these agents, contemporaneous data examining both pharmacokinetic (PK) properties and efficacy in parallel have not previously been available.

Materials and methods: In this single-center double-blind study, 18 subjects (ten female) were treated for a single migraine attack with frovatriptan $2.5 \mathrm{mg}$ or rizatriptan $10 \mathrm{mg}$. Plasma concentrations were measured predose and at 2, 4, 6, 12, 24, 48, and 72 hours after drug administration. The primary end point of this study was to evaluate the association between PK parameters and efficacy measures and recurrence rate. Secondary end points were pain-free and pain-relief episodes at 2 and 4 hours, recurrent episodes within 48 hours, and cumulative hazard of recurrence within 72 hours.

Results: At baseline, approximately $17 \%$ of patients had mild migraine, while $83 \%$ had moderate-severe migraine. Although the time to maximum concentration was similar for both drugs (2.7 versus 2.3 hours), the terminal half-life for frovatriptan was longer than rizatriptan (29.3 versus 3.2 hours, $P<0.0001$ ). The proportion of patients who were pain-free at 4 hours without rescue medication was higher in the frovatriptan-treated group, (38.9 versus 5.6\%, $P=0.045)$. The cumulative hazard of recurrence over $72 \mathrm{~h}$ was reduced by frovatriptan compared to rizatriptan-treated patients (log-rank test, $P=0.04$ ). Pain-free and pain-relief episodes for the study period were positively correlated with the concentration:maximum concentration $\left(\mathrm{C}_{\max }\right)$ ratio for frovatriptan $(r=0.52, P=0.028)$, but not rizatriptan. Recurrence rate was negatively correlated with the concentration: $\mathrm{C}_{\max }$ ratio for both frovatriptan $(r=-0.96, P=0.0024)$ and rizatriptan ( $r=-0.98, P=0.0004)$. Fewer adverse events were observed for frovatriptan compared to rizatriptan (one versus eight, $P=0.021$ ).

Conclusion: This pilot study indicates that a similar extent of initial pain relief is afforded by both triptans in migraine treatment. The longer duration of action of frovatriptan parallels and correlates with its PK profile.

Keywords: headache, migraine, pain, frovatriptan, rizatriptan, rescue medication

\section{Introduction}

Migraine is a common painful neurological disorder that affects millions of individuals worldwide. ${ }^{1}$ It is frequently underdiagnosed and undertreated, and typically characterized by recurrent attacks of moderate-to-severe headache lasting 4-72 hours. ${ }^{2}$ Other common symptoms are nausea, vomiting, photo- and/or phonophobia and neurological aura symptoms. ${ }^{2}$ Although nonpharmacological therapies have been shown to offer symptom relief in the pediatric population, ${ }^{3,4}$ current guidelines recommend the use 
of pharmacological therapies as first-line treatment for mildto-moderate migraine in adults. ${ }^{5}$

Triptans are a class of selective serotonin 5-hydroxytryptamine (5-HT) 1B/1D agonists with proven efficacy for the treatment of acute migraine. ${ }^{6}$ The early success of the parent drug of this class, sumatriptan, led to the development of other triptan compounds, with the aim of optimizing pharmacokinetic (PK) properties, efficacy, and safety in migraine treatment. ${ }^{7}$ Triptans differ markedly in terms of their PK and pharmacodynamic properties. Some triptans, such as sumatriptan, are considered fast-acting while also possessing greater risk of adverse events (AEs). In contrast, other triptans, such as frovatriptan, provide a more prolonged duration of action, with fewer associated side effects. ${ }^{6}$

Frovatriptan was specifically developed to exhibit a long duration of action combined with reduced potential for side effects and drug interactions. ${ }^{8-11}$ It is a 5-HT-receptor agonist that binds with high affinity to $5-\mathrm{HT}_{1 \mathrm{~B}}$ and $5-\mathrm{HT}_{1 \mathrm{D}}$ receptors, but unlike other triptans, frovatriptan has moderate affinity for $5-\mathrm{HT}_{7}$ receptors. ${ }^{12}$

A unique PK feature of frovatriptan is its long terminal elimination half-life of approximately 26 hours, unlike other triptans, which range from 3-6 hours. ${ }^{13}$ Frovatriptan does not inhibit or induce cytochrome P450 isoenzymes, and is only slightly bound to plasma proteins, thus exhibiting a low risk of PK drug interactions. ${ }^{14}$

Rizatriptan is also a selective serotonin $5-\mathrm{HT}_{1}(1 \mathrm{~B} / 1 \mathrm{D})$ agonist. ${ }^{15,16}$ It is rapidly absorbed, eliminated with a plasma half-life of approximately 2-3 hours, and is reported to induce rapid pain relief. ${ }^{15-17}$ Since the majority of rizatriptan is metabolized by monoamine oxidase A, cytochrome P450 inhibitors have minimal effects on the PK of rizatriptan. ${ }^{18}$

While both frovatriptan and rizatriptan have independently proven efficacy and tolerability for the treatment of acute migraine, ${ }^{11,16}$ only two head-to-head trials have actually compared the efficacy of these two triptans. ${ }^{19,20}$ Both studies were performed on Italian migraineurs (one of which was in females with migraine that was menstrually related), and both demonstrated similar antimigraine efficacy of the two triptans in terms of pain-free and pain-relief rates. However, the hazard of migraine recurrence after 48 hours was significantly lower with frovatriptan use in both studies. ${ }^{19,20}$ Migraine headache recurrence is an important clinical problem in migraine management. ${ }^{21}$ The mechanism of headache recurrence is not well understood, but it is thought to be linked with patient-specific physiological characteristics and PK properties of the drug used. ${ }^{22}$ A meta-analysis of more than 30 randomized, double-blind studies showed a strong inverse correlation between the frequency of migraine headache recurrence and half-lives of different triptans. ${ }^{22}$ The triptans with longer terminal elimination half-lives, namely eletriptan, naratriptan, and frovatriptan, had a lower incidence of headache recurrence. ${ }^{22}$

Although previous studies have independently compared the efficacy of these agents in head-to-head trials, this is the first study to specifically examine both PK properties and efficacy in real time and in parallel. Therefore, the primary objective of this study was to confirm previous data by evaluating the presence of a potential association between recurrence rates and $\mathrm{PK}$ parameters for two pharmacokinetically distinct triptans: frovatriptan and rizatriptan. Secondary measures included standard-efficacy parameters of pain-free and pain-relief-rates at 2 and 4 hours in addition to recurrence rates within 48 hours.

\section{Materials and methods Patients}

This pilot study was performed on patients in the Institute for Pharmacokinetic and Analytical Studies Clinic, Ligornetto, Switzerland between July 2008 and March 2010. Patients included both male and female subjects aged between 18 and 55 years, with a current history of migraine with or without aura, according to International Headache Society (IHS) 2004 criteria, and with at least one but no more than six migraine attacks per month in the 6 months prior to entering the study. ${ }^{2}$ Patients could not be enrolled in the study if they had: 1) uncontrolled hypertension; 2) ischemic heart disease; 3 ) cardiac arrhythmias or symptomatic WolffParkinson-White syndrome; 4) previous stroke or transient ischemic attack; 5) severe liver or renal impairment; 6) any other severe or disabling medical condition; 7) a history of alcohol, analgesic, or psychotropic drug abuse; 8) known hypersensitivity to study drugs; 9) previously demonstrated inadequate response to at least two triptans; 10) current use of propranolol or ergotamine (and its derivatives) as a prophylactic agent; 11) current use or use in the previous 2 weeks of monoamine oxidase inhibitors; 12) use of either test medication to treat any one of the last three episodes of migraine; and 13) other headaches that had lasted for more than 6 days (at the time of presentation for the study). Patients were permitted to continue with concomitant medications during the trial if prescribed for concomitant illnesses. Ideally, doses were kept stable during the study, but if a change in the concomitant medications and/or doses was considered essential for medical reasons, these measures were recorded in the case-report form and patient diary. 
Where possible, the dose of any prophylactic medication remained unchanged throughout the study period. During the blood-sampling time, paracetamol was preferred as a concomitant medication for the treatment of headache. Where indicated, emergency medication could be administered at any time during the trial. Pregnant women and breastfeeding mothers were excluded as well, while women with childbearing potential but not practicing an effective method of birth control were to be submitted to a pregnancy test, if clinically indicated. The study was approved by the independent institutional review boards of the study center, was performed in accordance with IHS guidelines, and was in compliance with the Declaration of Helsinki. Written informed consent was obtained from all patients prior to their inclusion into the study.

\section{Study design}

This was a double-blind, single-dose, balanced, two-sequence, two-period, two-treatment, randomized crossover design with at least 14 days of washout between the two treatments. Each patient received $2.5 \mathrm{mg}$ frovatriptan and $10 \mathrm{mg}$ rizatriptan in a balanced computer-generated randomized sequence (1:1), where frovatriptan was followed by rizatriptan or vice versa. Blinding was ensured by preparing identical capsules containing frovatriptan or rizatriptan. The duration of the study varied between subjects, depending on the interval between screening and the first migraine attack and between the two migraine attacks. However, patients were instructed to go to the clinic as soon as a migraine attack occurred (and to take study medication as soon as possible after migraine occurrence).

\section{Safety assessments}

The safety of medications administered during the trial was monitored by recording in the patient's diary all AEs occurring during each period of treatment. In addition, vital signs (respiratory rate, blood pressure, and heart rate) were measured and recorded at each visit (before and at end of study).

\section{Efficacy assessments}

Patients recorded the following information in paper diaries:

- date and time of onset of migraine

- date and time of treatment with study medication

- headache severity on a 4 -point scale $(0=$ none, $1=$ mild, $2=$ moderate, $3=$ severe) at the following times: 0 (time of treatment) and 2, 4, 6, 12, 24, 48, and 72 hours after treatment

- associated symptoms (nausea, vomiting, photophobia, phonophobia) on the same 4-point scale and at the same times.

\section{Pharmacokinetic assessments}

Plasma was collected for determination of rizatriptan and frovatriptan concentrations at the following times: predose, and at 2, 4, 6, 8, 12, 24, 48, and 72 hours postdose. Deviations in blood-sampling times were taken into account in the calculation of PK parameters. A $10 \mathrm{~mL}$ blood sample was collected from an indwelling catheter and allowed to clot at $2^{\circ} \mathrm{C}-8^{\circ} \mathrm{C}$ without any anticoagulant. Plasma was separated by centrifugation at $4^{\circ} \mathrm{C}$ and $1,000 \mathrm{~g}$ for 15 minutes. Plasma samples were checked and stored in freezers at $-80^{\circ} \mathrm{C} \pm 10^{\circ} \mathrm{C}$ pending the assay. All blood/ plasma samples were identified by labels bearing the study code, the subject number, the treatment period, the time of blood collection, and the aliquot identification. Frovatriptan and rizatriptan plasma concentrations were measured by fully validated liquid chromatography-mass spectrometry methods.

\section{Statistical analysis}

The sample-size calculation of 25 subjects was based on a statistical power of $80 \%$, a significance level of $5 \%$, and a variation coefficient of the main efficacy variables (painfree and pain-relief rates at $2 \mathrm{~h}$ ) equal to $25 \%$. The primary end point of interest was the correlation between plasma concentration of each triptan (and more specifically the concentration:maximum concentration $\left(\mathrm{C}_{\max }\right)$ ratio and the pain-free and pain-relief rates at each time point. Secondary efficacy parameters were:

- pain-free rates at 2 and 4 hours

- pain-relief rates at 2 and 4 hours

- recurrence rates at 24,48 , and 72 hours

- associated symptoms (nausea, vomiting, photophobia, phonophobia) at each time point

- rescue-medication use

- drug preference (frovatriptan, rizatriptan, or no preference). ${ }^{20}$

Pain-free, pain-relief and pain-recurrence were defined according to IHS criteria. ${ }^{2}$

All data are presented as means \pm standard deviation or percentages. All statistical analysis was performed with the Stata statistical software package, version 10 (StataCorp, College Station, TX, USA) or InStat (GraphPad Software, La Jolla, CA, USA). Nonparametric continuous variables were compared by the Mann-Whitney test. Comparisons between two groups with normally distributed variables were analyzed by unpaired $t$-tests. Categorical variables were analyzed by $\chi^{2}$ tests. Univariate correlations (eg, between PK parameters and efficacy measures) were assessed by Pearson's 
correlation coefficient $(r)$. Where comparisons were made, quoted $P$-values were two-tailed.

The $P K$ parameters $\mathrm{C}_{\max }$ and time to $\mathrm{C}_{\max }\left(\mathrm{T}_{\max }\right)$ were read directly using WinNonlin software (Pharsight, Mountain View, CA, USA). Terminal half-life $\left(t_{1 / 2}\right)$ values were determined by extrapolating the terminal portion of the curve. Area under the plasma concentration-time curve (AUC) values were calculated from individual concentration-time data using WinNonlin software. The AUC from the time of dosing to the last quantifiable concentration was calculated by means of the linear trapezoidal rule. Extrapolation to infinity was obtained by dividing the last quantifiable concentration by the terminal elimination-rate constant and adding this result to $\mathrm{AUC}_{\mathrm{t}}$. The concentration: $\mathrm{C}_{\max }$ ratio was calculated by dividing the instantaneous plasma concentration by the observed $\mathrm{C}_{\max }$. Univariate regression analysis was performed between selected PK parameters (eg, $\mathrm{C}_{\text {max }}, \mathrm{AUC}$, concentration: $\mathrm{C}_{\max }$ ratio) and efficacy parameters (eg, painfree, pain-relief, combined pain-free and pain-relief rates). Kaplan-Meier curves were used to determine the cumulative hazard of recurrence over 72 hours. A $P$-value of $<0.05$ was considered statistically significant. Bonferroni post hoc testing was applied to adjust for potential multiplicity.

\section{Results}

\section{Baseline demographic and clinical characteristics}

From a total of 25 screened and randomized subjects, seven were excluded from the analyses as they did not experience a migraine attack within 3 months, as planned in the protocol. These subjects were excluded from the study without having taken any study treatment, and were not replaced. The remaining 18 subjects completed both treatment groups and are included in the PK, safety, and efficacy analyses.

Patient disposition is summarized in Figure 1. Baseline clinical characteristics for all 18 patients included in the study are shown in Table 1 . The majority of patients (83.3\%)

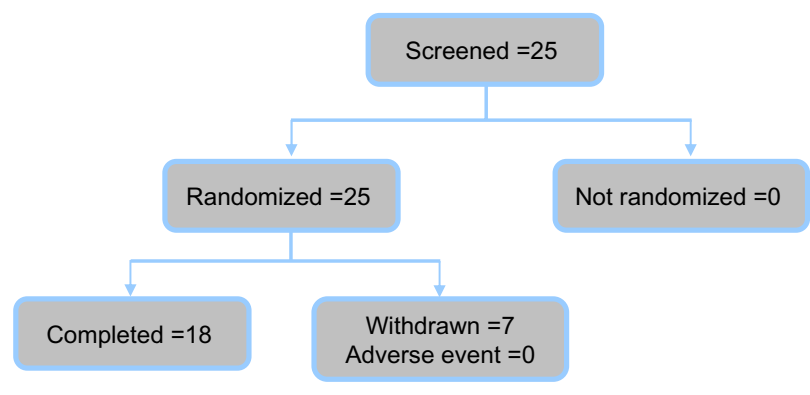

Figure I Patient disposition.
Table I Baseline clinical characteristics

\begin{tabular}{|c|c|c|c|}
\hline $\begin{array}{l}\text { Clinical } \\
\text { characteristics }\end{array}$ & $\begin{array}{l}\text { All subjects } \\
n=18\end{array}$ & $\begin{array}{l}\text { Sequence } 1 \\
n=18\end{array}$ & $\begin{array}{l}\text { Sequence } 2 \\
n=18\end{array}$ \\
\hline \multicolumn{4}{|l|}{ General } \\
\hline Female, n (\%) & $10(55.5)$ & $10(55.5)$ & $10(55.5)$ \\
\hline Age (years) & $39.4 \pm 7.8$ & $39.4 \pm 7.8$ & $39.4 \pm 7.8$ \\
\hline Height (cm) & $168.5 \pm 7.7$ & $168.5 \pm 7.7$ & $168.5 \pm 7.7$ \\
\hline Body weight (kg) & $69.7 \pm 77.6$ & $69.7 \pm 77.6$ & $69.7 \pm 77.6$ \\
\hline $\mathrm{DBP}(\mathrm{mmHg})$ & $79.2 \pm 6.7$ & $76.9 \pm 6.4$ & $74.3 \pm 5.5$ \\
\hline $\mathrm{SBP}(\mathrm{mmHg})$ & $119.4 \pm 10.3$ & II $5.89 \pm 7.4$ & $113.44 \pm 6.3$ \\
\hline \multicolumn{4}{|l|}{ Migraine severity } \\
\hline Mild intensity, n (\%) & $3(16.6)$ & $2(I I . I)$ & $2(11.1)$ \\
\hline $\begin{array}{l}\text { Moderate intensity, } \\
\mathrm{n}(\%)\end{array}$ & $12(66.6)$ & I 4 (77.8) & $12(66.6)$ \\
\hline Severe intensity, $\mathrm{n}(\%)$ & $3(16.6)$ & $2(11.1)$ & $4(22.2)$ \\
\hline
\end{tabular}

Note: Data presented as means \pm standard deviation or $n(\%)$.

Abbreviations: DBP, diastolic blood pressure; SBP, systolic blood pressure.

had moderate-to-severe migraine at the start of treatment. Seven patients (38.8\%) were taking at least one concomitant medication at screening and during both treatment periods. In addition to baseline characteristics, vital parameters were also measured prior to undertaking the study, at the visit between the two study periods (switch after first treatment), and at the end of the study (after second treatment) (Table 1).

\section{Pharmacokinetic analysis}

Mean plasma concentrations of frovatriptan and rizatriptan over the study period are shown in Figure 2A, and the

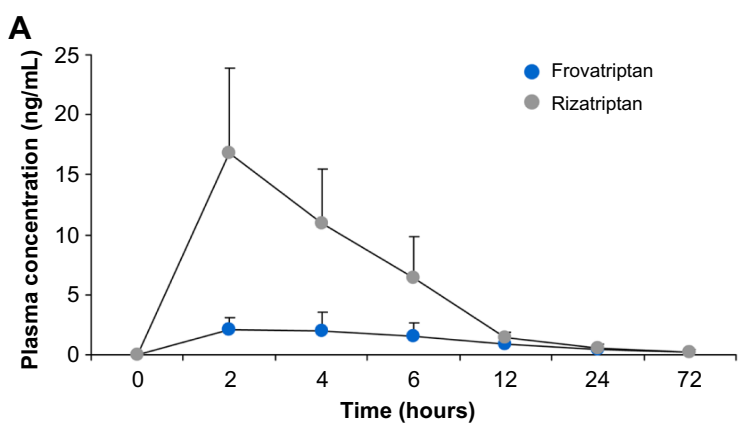

B

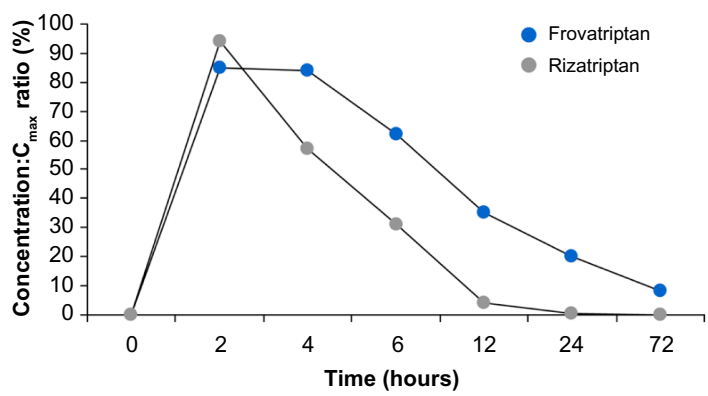

Figure 2 (A and B) Pharmacokinetic profile and plasma levels of frovatriptan and rizatriptan.

Notes: (A) Plasma levels $(\mathrm{ng} / \mathrm{mL})$ and concentration: $\mathrm{C}_{\max }$ ratio and $(\mathbf{B})$ of the two drugs over the study period. Data presented as means or means \pm standard deviation. Abbreviation: $\mathrm{C}_{\max }$, maximum observed plasma concentration. 
observed and calculated PK parameters are shown in Table 2. The concentration: $\mathrm{C}_{\max }$ ratio (\%) peaked at the 2-hour time point for both drugs and shifted forward 2 hours (faster absorption rate) for frovatriptan compared to rizatriptan (Figure $2 \mathrm{~B}$ ). $\mathrm{T}_{\max }$ was similar for frovatriptan and rizatrip$\tan$ (2.7 versus 2.3 hours, respectively), whereas the $t_{1 / 2}$ for frovatriptan was significantly longer than rizatriptan (29.3 versus 3.2 hours, $P<0.0001$ ) (Table 2). Subanalysis of PK parameters by sex was also performed, and this revealed significantly higher $\mathrm{C}_{\text {max }}(3.2 \pm 1.5$ versus $1.49 \pm 0.55 \mathrm{ng} / \mathrm{mL}$, $P=0.009)$ and $t_{1 / 2}(32.8 \pm 7.8$ versus $24.9 \pm 7.7$ hours, $P=0.048)$ values for frovatriptan in females compared to male subjects. Sex-specific differences in PK parameters were also observed for rizatriptan, but did not attain statistical significance.

\section{Pain-free and pain-relief rates and migraine recurrence}

Pain-free, pain-relief, and recurrence rates are summarized in Table 3. Although no differences were observed in pain-free rates at 2 hours, frovatriptan was more effective than rizatrip$\tan$ at 4 hours ( 38.9 versus $5.6 \%, P=0.045$ ). Pain-relief rates were similar between frovatriptan and rizatriptan. In contrast, the number of recurrent episodes was significantly higher for rizatriptan- compared to frovatriptan-treated patients, attaining statistical significance at 48 hours $(55.6 \%$ versus $27.8 \%$, $P=0.046$ ). This finding was confirmed by the observation of a significantly lower cumulative hazard of migraine recurrence $(P=0.04)$ over 72 hours in patients treated with frovatriptan (Figure 3). No difference was observed between male and female subjects for pain-free or pain-relief rates or rate of migraine recurrence.

\section{Association between pharmacokinetic profile and pain-free or recurrence rate}

The univariate regression analysis performed between the concentration: $\mathrm{C}_{\max }$ ratio (\%) and pain-free/pain-relief parameters (Figure 4) showed a positive correlation between

Table 2 Pharmacokinetic parameters

\begin{tabular}{llcl}
\hline Parameter & $\begin{array}{l}\text { Frovatriptan } \\
\mathbf{n}=\mathbf{1 8}\end{array}$ & $\begin{array}{l}\text { Rizatriptan } \\
\mathbf{n}=1 \mathbf{8}\end{array}$ & P-value \\
\hline $\mathrm{C}_{\max }(\mathrm{ng} / \mathrm{mL})$ & $2.44 \pm \mathrm{I} .5$ & $15.7 \pm 6.1$ & $<0.0001$ \\
$\mathrm{~T}_{\max }$ (hours) & $2.7 \pm 0.95$ & $2.3 \pm \mathrm{I}$ & 0.25 \\
$\mathrm{AUC}_{\mathrm{t}}(\mathrm{ng} / \mathrm{mL} / \mathrm{h})$ & $40.8 \pm 28$ & $72.1 \pm 26$ & 0.0014 \\
$\mathrm{AUC}_{\infty}(\mathrm{ng} / \mathrm{mL} / \mathrm{h})$ & $50.7 \pm 33.6$ & $73 \pm 26$ & 0.033 \\
$t_{1 / 2}$ (hours) & $29.3 \pm 8.5$ & $3.2 \pm 2.9$ & $<0.0001$ \\
\hline
\end{tabular}

Abbreviations: $C_{\max }$, maximum observed plasma concentration; $T_{\max }$, time to reach $\mathrm{C}_{\max } ; \mathrm{AUC}$, area under the plasma concentration-time curve from zero to time $\mathrm{t}$ : $A \cup C_{\infty}, A \cup C$ from zero to infinity; $t_{1 / 2}$, plasma half-life.
Table 3 Secondary efficacy end points in the two study treatment groups

\begin{tabular}{|c|c|c|c|}
\hline Efficacy end points & $\begin{array}{l}\text { Frovatriptan } \\
n=18\end{array}$ & $\begin{array}{l}\text { Rizatriptan } \\
n=18\end{array}$ & $P$-value \\
\hline Pain-free episodes at 2 hours* & $3(16.7)$ & $2(11.1)$ & NS \\
\hline Pain-free episodes at 4 hours* & $7(38.9)$ & I (5.6) & 0.045 \\
\hline Pain-relief episodes at 2 hours & $8(44.4)$ & II (6I.I) & NS \\
\hline Pain-relief episodes at 4 hours & II (6I.I) & $13(72.2)$ & NS \\
\hline $\begin{array}{l}\text { Recurrent episodes within } \\
24 \text { hours }\end{array}$ & $2(11.1)$ & $6(33.3)$ & NS \\
\hline $\begin{array}{l}\text { Recurrent episodes within } \\
36 \text { hours }\end{array}$ & $4(22.2)$ & $8(44.4)$ & NS \\
\hline $\begin{array}{l}\text { Recurrent episodes within } \\
48 \text { hours }\end{array}$ & $5(27.8)$ & $10(55.6)$ & 0.046 \\
\hline
\end{tabular}

Note: *Without rescue medication.

Abbreviation: NS, not significant.

frovatriptan concentration: $\mathrm{C}_{\max }$ ratio (\%) and the proportion of patients that were either pain free or experienced pain relief over the entire study period (Figure 4A). No such correlation was seen for rizatriptan (Figure 4B). We next directly correlated cumulative recurrence over the study period with the concentration: $\mathrm{C}_{\max }$ ratio, and observed a significant inverse correlation for both frovatriptan- $(r=-0.96$, $P=0.0028)$ and rizatriptan-treated $(r=-0.98, P=0.0004)$ groups (Figure 4C). While recurrence rates remained similar at higher concentration: $\mathrm{C}_{\max }$ ratios of $20 \%-90 \%$, as highlighted in the figure, the curves markedly diverge at a concentration: $\mathrm{C}_{\max }$ ratio of $<20 \%$, whereby cumulative recurrence rate nears $60 \%$ in patients treated with frovatriptan and reaches $100 \%$ in those treated with rizatriptan (Figure 4C).

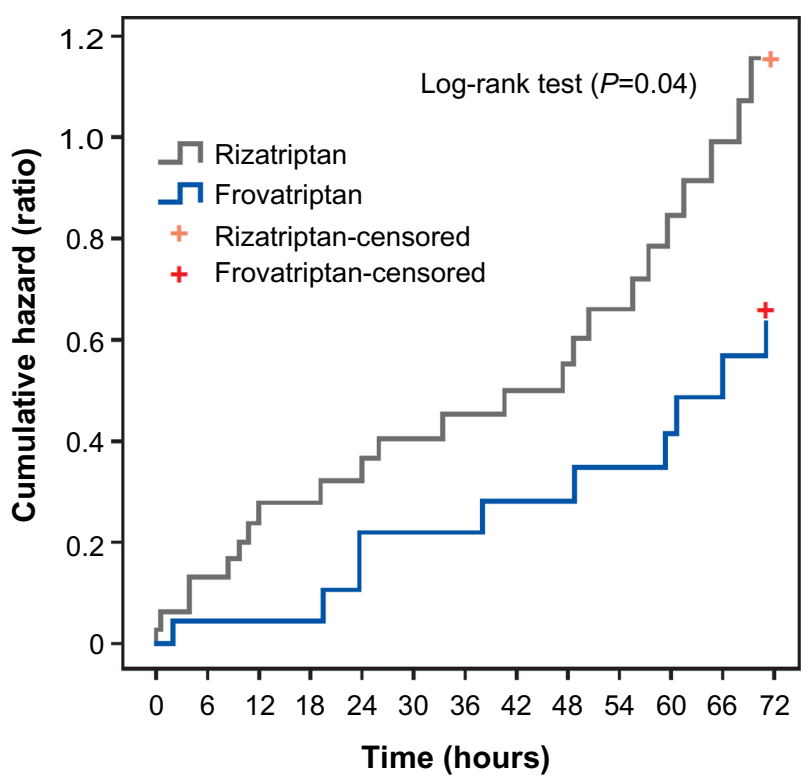

Figure 3 Kaplan-Meier plot of cumulative hazard of migraine recurrence over 72 hours in patients treated with frovatriptan compared to rizatriptan.

Note: The $P$-value represents a significant difference between the two treatment groups. 
A

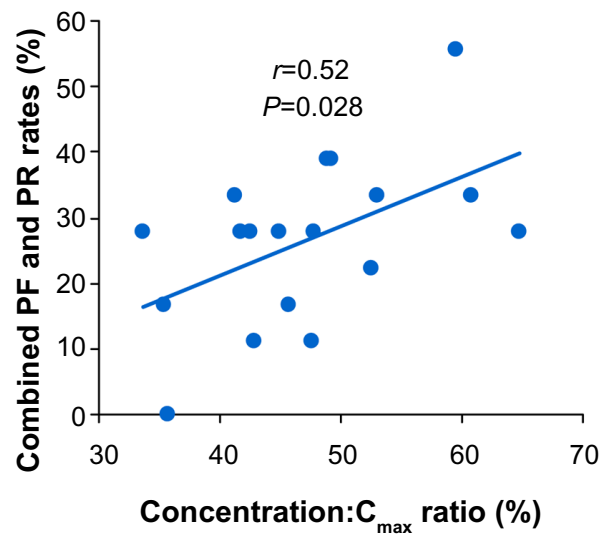

B

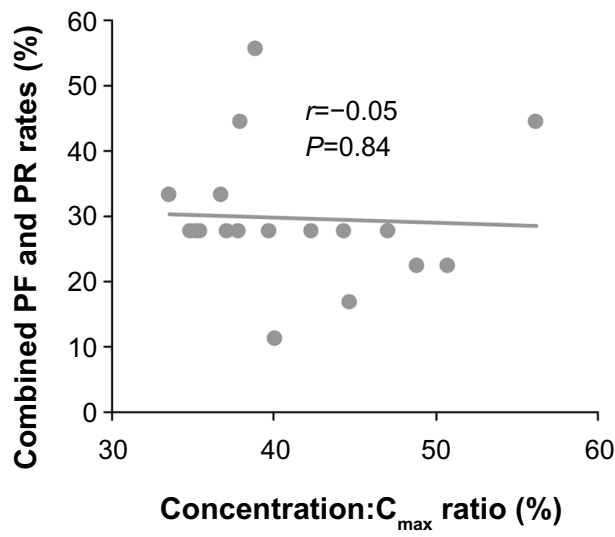

C

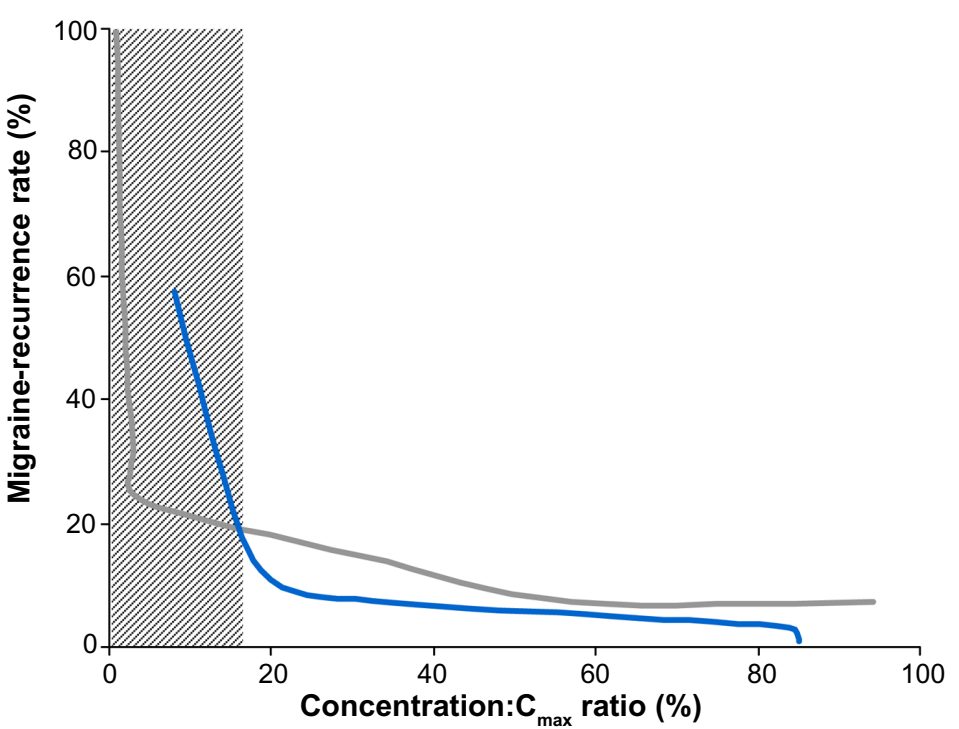

Figure 4 (A-C) Association between pharmacokinetic profile and effect of treatment on pain-free (PF), pain-relief (PR) rate, and rate of migraine recurrence. Notes: (A) Univariate regression plot of concentration: $\mathrm{C}_{\max }$ ratio (\%); (B) proportion of patients who were PF or had PR with frovatriptan or rizatriptan. (C) Univariate regression curves showing negative correlation between migraine-recurrence rate and concentration: $\mathrm{C}_{\max }$ ratio for frovatriptan- and rizatriptan-treated patients. Regression coefficients $(r)$ and level of statistical significance are indicated.

Abbreviation: $\mathrm{C}_{\max }$, maximum observed plasma concentration.

This analysis was extended to other PK parameters, but did not reveal any additional associations.

\section{Migraine-associated symptoms}

Both drugs gradually reduced migraine-related symptoms, with no difference observed between treatments (Figure 5A-D). Although differences were observed at baseline for nausea and photophobia, these differences were not statistically significant. A significant reduction in patients experiencing nausea $(P=0.045)$, photophobia $(P<0.001)$, and phonophobia $(P<0.001)$ was observed at 72 hours for both drugs compared to baseline (Figure 5B-D).

\section{Drug preference}

No significant difference was observed in the proportion of patients who preferred frovatriptan versus rizatriptan (five subjects [27.8\%] versus six subjects [33.3\%]). Seven (38.9\%) patients had no preference for either drug.

\section{Rescue medication}

Ten patients $(55.5 \%)$ required concomitant medication to treat their migraine when the study drug provided insufficient relief. Five patients received single-medication treatment, whereas the remaining five patients required two or more doses of the same or two or more different medications. 
Frovatriptan

Rizatriptan

A

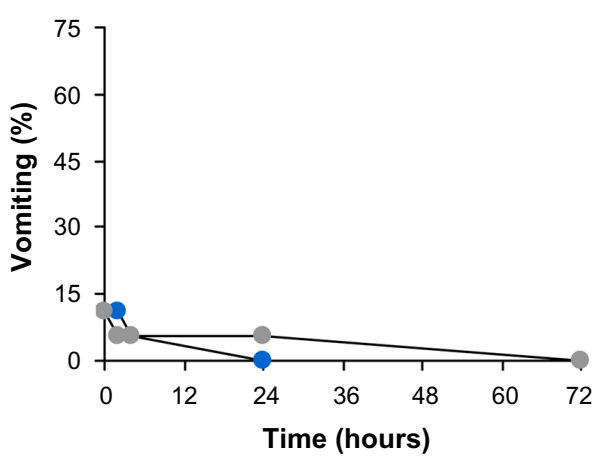

C

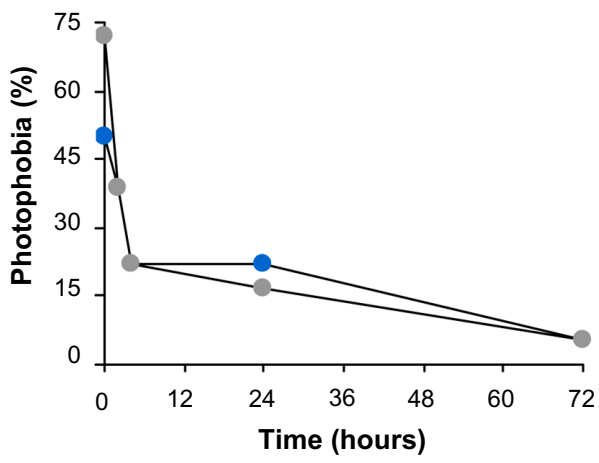

B

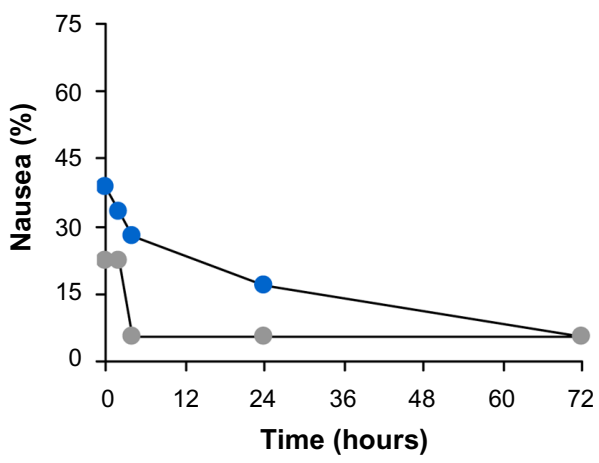

D

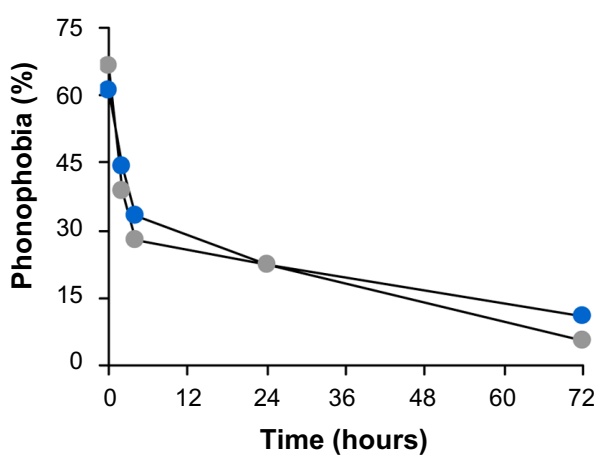

Figure 5 (A-D) Frequency of different migraine symptoms after treatment with frovatriptan or rizatriptan. Note: Data presented as percentages.

In total, 31 different concomitant medications were taken among the patients, the most frequent being paracetamol $(\mathrm{n}=11)$, caffeine $(\mathrm{n}=6)$, and indomethacin $(\mathrm{n}=5)$. Other medications included prochlorperazine $(n=4)$, nimesulide $(n=2)$, meclizine $(\mathrm{n}=1)$, pyridoxine $(\mathrm{n}=1)$, and propyphenazone $(n=1)$. Twenty different migraine attacks were treated using combinations of rescue medication on top of frovatriptan or rizatriptan. The frequency of rescue medication was lower in patients during frovatriptan treatment (seven of 20) compared to rizatriptan treatment (13 of 20), this difference just failing to reach statistical significance $(P=0.058)$.

\section{Safety}

A total of nine AEs were reported by nine subjects during the study (Table 4). One event was reported during frovatriptan treatment compared to eight during rizatriptan treatment

Table 4 Adverse events by severity reported for frovatriptan and rizatriptan

\begin{tabular}{|c|c|c|c|c|c|c|c|c|}
\hline \multirow[t]{2}{*}{ AEs } & \multicolumn{2}{|l|}{ Mild } & \multicolumn{2}{|c|}{ Moderate } & \multicolumn{2}{|c|}{ Severe } & \multicolumn{2}{|c|}{ Total AEs } \\
\hline & Frov & $\mathbf{R i z}$ & Frov & $\mathbf{R i z}$ & Frov & $\mathbf{R i z}$ & Frov & Riz \\
\hline Sore throat & 0 & I & 0 & I & 0 & 0 & 0 & 2 \\
\hline Asthenia & 0 & 0 & 0 & 0 & 0 & I & 0 & I \\
\hline Precordial pain with respiration & 0 & 1 & 0 & 0 & 0 & 0 & 0 & I \\
\hline Respiratory difficulty & 0 & 0 & 0 & 0 & I & 0 & I & 0 \\
\hline Dizziness & 0 & I & 0 & 1 & 0 & 0 & 0 & 2 \\
\hline Pollen allergy & 0 & I & 0 & 0 & 0 & 0 & 0 & 1 \\
\hline Vertigo & 0 & I & 0 & 0 & 0 & 0 & 0 & I \\
\hline Total AEs reported & 0 & $5^{*}$ & 0 & 2 & I & I & I & $8^{*}$ \\
\hline Total patients (\%) & 0 & 27.8 & 0 & II.I & 5.5 & 5.5 & 5.5 & 44.4 \\
\hline
\end{tabular}

Note: $* P<0.05$ (frovatriptan- versus rizatriptan-treated groups, $\chi^{2}$ test). 
(relative risk 2.4, confidence interval 1.39-4.1; $P=0.021$ ). The majority of AEs (seven events) were of mild or moderate intensity, and no serious AEs were reported during the study. There was a small decrease in respiratory rate in both treatment groups during treatment, but no clinically-relevant change in any of the other vital signs (heart rate, blood pressure, or temperature) in either treatment group.

\section{Discussion}

The present head-to-head trial compared the PK and clinical activity of the two triptans frovatriptan and rizatriptan in 18 patients suffering from migraine and treated with these two drugs according to a double-blind, two-way, crossover design, with a 14-day washout period. Although the therapeutic efficacy of frovatriptan has been directly compared to other triptans, ${ }^{19,20,23,24}$ including rizatriptan, ${ }^{19,20}$ this was the first study to specifically examine both the PK profile and therapeutic efficacy of two pharmacokinetically distinct triptans in parallel.

PK analysis revealed similar kinetics for the concentration: $\mathrm{C}_{\max }$ ratio during the early phase, while both drugs differed substantially over later time points (4-72 hours). A marked difference was observed in the $t_{1 / 2}$ between frovatriptan (29.3 hours) compared to rizatrip$\tan$ (3.24 hours). These two distinct PK profiles are already established. ${ }^{8}$ Frovatriptan has been shown to have a $t_{1 / 2}$ of approximately 26 hours, which was not affected by sex, dose, or mode of administration. ${ }^{13}$ Other studies have shown similar $t_{1 / 2}$ for frovatriptan, which varied slightly due to age and/or sex. ${ }^{25}$ In contrast, rizatriptan has been shown to have an extremely fast plasma elimination $t_{1 / 2}$, typically ranging between 2 and 2.5 hours, corroborating findings observed in the present report. ${ }^{15,18}$

Frovatriptan has distinctive pharmacologic features compared with other triptans, which make it particularly well suited to patients with prolonged migraines and those suffering migraine recurrence..$^{9,10,26}$ In addition, due to the lack of inhibitory or inducing effects on cytochrome P450 isoenzymes, frovatriptan retains a low risk of drug interactions, and dosage adjustment is unlikely to be warranted. This is particularly relevant, since combination treatment of frovatriptan with analgesic drugs is sometimes required. Although the number of subjects in our study was small, our safety analysis replicated the findings in other studies in which frovatriptan exhibited a favorable tolerability profile that was often better than comparator triptans. ${ }^{11}$ As expected, both drugs also gradually decreased migraine-related symptoms, as was also previously observed..$^{20}$
It is worth noting that sex-specific differences were observed for frovatriptan, for some PK parameters examined, but not for rizatriptan. Female subjects had significantly higher $\mathrm{C}_{\max }$ and $t_{1 / 2}$ values compared to male subjects. These sex-specific effects have also been observed in previous findings in healthy adults and adolescent migraineurs. ${ }^{13,25}$ This difference has mainly been attributed to greater bioavailability in females compared to male subjects, in addition to a higher volume of distribution and clearance in males. ${ }^{11,13,25}$ Regardless, these PK sex-specific differences for frovatriptan do not have any clinically significant effect, in terms of efficacy or safety, since frovatriptan has an extremely wide therapeutic window, and thus no dosage adjustment was deemed necessary. ${ }^{13}$ Supporting this, our findings also demonstrated that the activity of frovatriptan in terms of clinical benefit was similar between males and females.

Comparing efficacy of the two drugs in the present study, we did not see any difference between the two agents at 2 hours, but did observe a higher pain-free rate at 4 hours. Other head-to-head trials comparing frovatriptan to another triptan in migraineurs have observed similar extent of benefit afforded by frovatriptan. ${ }^{19,20,23,24}$ Furthermore, pain-relief rates at 2 hours (ranging between $40 \%$ and $50 \%$ ) were similar for both drugs and also corroborate previous findings. ${ }^{19,20,23,24}$ These data, collectively, indicate that the extended duration of effect associated with frovatriptan does not come at the expense of a slower onset of action. We also demonstrated that the frequency of pain-free episodes and pain-relief rates were directly correlated with the PK profile of frovatriptan. In addition to providing acute benefit, in terms of painfree episodes, frovatriptan also significantly reduced recurrent episodes within 48 hours compared to rizatriptan. Recurrent episodes within 48 hours have typically lower incidence, ranging from $20 \%$ to $30 \%$ for frovatriptan compared to $43 \%$ for rizatriptan, ${ }^{20} 44 \%$ for almotriptan, ${ }^{23}$ and $50 \%$ for zolmitriptan. ${ }^{24}$ The numerical differences in recurrence rates at 24 and 48 hours also suggest that beneficial effects on recurrence might also have been seen with frovatriptan with a larger sample size.

The difference in $\mathrm{PK}$ profile as seen by the concentration: $\mathrm{C}_{\max }$ ratio (\%) for frovatriptan and rizatriptan from 4 to 72 hours was shown to correlate negatively with recurrence rate. Our findings reinforce the association observed between recurrence rate and PK activity $\left(t_{1 / 2}\right)$ of various triptans, previously documented by Géraud et al, ${ }^{22}$ but a small sample size hampered the strength of our analysis. Although our findings indicated that PK activity may be an important factor in determining frequency of migraine 
recurrence, this continues to be an extremely complex physiological phenomenon that cannot be explained solely by $\mathrm{PK}$ activity. ${ }^{22}$ While it is important not to overestimate the clinical importance of different PK profiles of triptans, ${ }^{27}$ previous evidence, ${ }^{22}$ including the present report, points toward the need for additional trials with greater sample sizes and with well-defined end points.

\section{Study limitations}

The main limitation of the present study was the small sample size. This study was slightly underpowered due to the exclusion of seven patients, as they had not experienced a migraine attack within 3 months, as planned in the protocol. A larger sample size would have permitted a multivariate approach to determine more precisely the cause-effect relationship between PK profile and drug effect (ie, antimigraine outcome measures). Regardless, even with the small sample size, secondary efficacy measures (eg, pain-free and pain-relief rates and rate of recurrence) were similar to other previously published studies. ${ }^{19,20,23,24}$ Additional time points for secondary measures and a slightly longer follow-up period would have been desired. Although the predominant analgesic effect was attributed to triptan use, it should also be taken into account that in some subjects treatment with anti-inflammatory drugs was required, as planned in the protocol, which may have contributed in part to pain relief. Regardless, the double-blind, randomized, crossover design controlled for these and other potential confounders. A second potential weakness of the present study was the fact that PK parameters were measured from plasma samples and not whole blood. Since rizatriptan (and subsequent PK parameters) is normally assayed from plasma, ${ }^{15,18}$ we also specifically assayed frovatriptan in the same manner, since this was a crossover design. However, this could be particularly relevant for frovatriptan, since it is recognized to bind to red blood cells (60\% reversibly bound at steady states), thus potentially compromising assays performed exclusively in plasma. ${ }^{28,29}$ However, values for different PK parameters measured in the present study were well within the normal range for those described using whole blood, ${ }^{13,25}$ arguing that the plasma versus whole blood difference (particularly for frovatriptan) does not appear to cause an observable effect, at least at the doses used in the present study.

Finally, we included a limited PK sampling schedule in our study with the intention of correlating plasma levels and efficacy at selected time points. This meant that we were unable to fully define the PK profiles of both drugs, and particularly for rizatriptan, the shape of our observed curve suggested that $\mathrm{T}_{\text {max }}$ would have occurred earlier than our first sample time ( 2 hours). While this is a limitation for a pure PK analysis, it does not necessarily undermine the conclusions we have drawn from the PK efficacy correlations. Indeed, an earlier $\mathrm{T}_{\text {max }}$ and higher true $\mathrm{C}_{\max }$ for rizatriptan would result in even lower concentration: $\mathrm{C}_{\max }$ ratios for rizatriptan and so magnify further the differences between the two agents.

\section{Conclusion}

In conclusion, this is the first study to specifically examine both the PK profile and therapeutic efficacy of two pharmacokinetically distinct triptans in parallel. Findings from the present pilot study confirmed the expected clinical activity of the two drugs on a target population and confirm previously published PK characteristics. Although both frovatriptan and rizatriptan displayed similar PK activity in the early phase ( $0-4$ hours), marked differences in activity were observed in the intermediate period after dosing, particularly 4-24 hours, where the longer $t_{1 / 2}$ of frovatriptan and the persistence of its active plasma concentrations may explain its better performance at later time points. This time period (4-24 hours) coincided with improved efficacy by frovatriptan in terms of reduction of migraine attacks and reduction of migraine recurrence compared to rizatriptan. PK parameters were also found to be associated with pain-free and pain-relief episodes, in addition to migraine recurrence. Results from the present trial support the use of frovatriptan over rizatriptan, particularly in patients suffering from relapse and/or migraines of longer duration. Patient-reported AEs and drug-related AEs were lower in patients treated with frovatriptan compared to rizatriptan. A further trial with a larger pool of patients will help confirm the results obtained in the present study.

\section{Acknowledgments}

The present study was supported by Menarini International Operations Luxembourg SA, Luxembourg. Dr Antonio Marzo (IPAS, SA, Switzerland) and Dr Colin Gerard Egan (Primula Multimedia SRL, Pisa, Italy) performed statistical analysis. The authors gratefully thank Dr Dario Zava (Istituto Lusofarmaco d'Italia, Milan, Italy) and Dr Steve Pawsey (Vernalis Group, UK) for critically revising the manuscript.

\section{Disclosure}

CGE and SM (on behalf of Primula Multimedia) received fees for manuscript preparation. LS declares no relevant financial interests. 


\section{References}

1. Lipton RB. Chronic migraine, classification, differential diagnosis, and epidemiology. Headache. 2011;51 Suppl 2:77-83.

2. Headache Classification Subcommittee of the International Headache Society. The International Classification of Headache Disorders. 2nd ed. Cephalalgia. 2004;24 Suppl 1:9-160.

3. Bellini B, Arruda M, Cescut A, et al. Headache and comorbidity in children and adolescents. J Headache Pain. 2013;14:79.

4. Jacobs H, Gladstein J. Pediatric headache: a clinical review. Headache. 2012;52(2):333-339.

5. Silberstein SD, Holland S, Freitag F, Dodick DW, Argoff C, Ashman E. Evidence-based guideline update: pharmacologic treatment for episodic migraine prevention in adults: report of the Quality Standards Subcommittee of the American Academy of Neurology and the American Headache Society. Neurology. 2012;78(17):1337-1345.

6. Loder E. Triptan therapy in migraine. $N$ Engl J Med. 2010;363(1): 63-70.

7. Cady R, Schreiber C. Sumatriptan: update and review. Expert Opin Pharmacother. 2006;7(11):1503-1514.

8. Tfelt-Hansen P, De Vries P, Saxena PR. Triptans in migraine: a comparative review of pharmacology, pharmacokinetics and efficacy. Drugs. 2000;60(6):1259-1287.

9. Balbisi EA. Frovatriptan succinate, a 5-HT1B/1D receptor agonist for migraine. Int J Clin Pract. 2004;58(7):695-705.

10. Kelman L. Review of frovatriptan in the treatment of migraine. Neuropsychiatr Dis Treat. 2008;4(1):49-54.

11. Sanford M. Frovatriptan: a review of its use in the acute treatment of migraine. CNS Drugs. 2012;26(9):791-811.

12. Comer MB. Pharmacology of the selective 5-HT(1B/1D) agonist frovatriptan. Headache. 2002;42 Suppl 2:S47-S53.

13. Buchan P, Keywood C, Wade A, Ward C. Clinical pharmacokinetics of frovatriptan. Headache. 2002;42 Suppl 2:S54-S62.

14. Buchan P, Wade A, Ward C, Oliver SD, Stewart AJ, Freestone S. Frovatriptan: a review of drug-drug interactions. Headache. 2002; 42 Suppl 2:S63-S73.

15. Goldberg MR, Lee Y, Vyas KP, et al. Rizatriptan, a novel 5-HT1B/1D agonist for migraine: single- and multiple-dose tolerability and pharmacokinetics in healthy subjects. J Clin Pharmacol. 2000;40(1): 74-83.

16. Mannix LK. A review of the 5-HT1B/1D agonist rizatriptan: update on recent research and implications for the future. Expert Opin Pharmacother. 2008;9(6):1001-1011.
17. Ferrari MD, Goadsby PJ, Roon KI, Lipton RB. Triptans (serotonin, 5-HT1B/1D agonists) in migraine: detailed results and methods of a meta-analysis of 53 trials. Cephalalgia. 2002;22(8):633-658.

18. Vyas KP, Halpin RA, Geer LA, et al. Disposition and pharmacokinetics of the antimigraine drug, rizatriptan, in humans. Drug Metab Dispos. 2000;28(1):89-95.

19. Savi L, Omboni S, Lisotto C, et al. Efficacy of frovatriptan in the acute treatment of menstrually related migraine: analysis of a double-blind, randomized, cross-over, multicenter, Italian, comparative study versus rizatriptan. J Headache Pain. 2011;12(6):609-615.

20. Savi L, Omboni S, Lisotto C, et al. A double-blind, randomized, multicenter, Italian study of frovatriptan versus rizatriptan for the acute treatment of migraine. J Headache Pain. 2011;12(2):219-226.

21. Lipton RB, Goadsby PJ, Sawyer JP, Blakeborough P, Stewart WF. Migraine: diagnosis and assessment of disability. Rev Contemp Pharmacother. 2000;11(2):63-73.

22. Géraud G, Keywood C, Senard JM. Migraine headache recurrence: relationship to clinical, pharmacological, and pharmacokinetic properties of triptans. Headache. 2003;43(4):376-388.

23. Bartolini M, Giamberardino MA, Lisotto C, et al. A double-blind, randomized, multicenter, Italian study of frovatriptan versus almotriptan for the acute treatment of migraine. J Headache Pain. 2011;12(3): 361-368.

24. Tullo V, Allais G, Curone M, et al. Frovatriptan versus zolmitriptan for the acute treatment of migraine with aura: a subgroup analysis of a double-blind, randomized, multicenter, Italian study. Neurol Sci. 2012;33 Supp1 1:S61-S64.

25. Elkind AH, Wade A, Ishkanian G. Pharmacokinetics of frovatriptan in adolescent migraineurs. J Clin Pharmacol. 2004;44(10):1158-1165.

26. Goldstein J. Frovatriptan: a review. Expert Opin Pharmacother. 2003;4(1):83-93.

27. Ferrari A, Tiraferri I, Neri L, Sternieri E. Why pharmacokinetic differences among oral triptans have little clinical importance: a comment. J Headache Pain. 2011;12(1):5-12.

28. Electronic Medicines Compendium. Migard (frovatriptan succinate monohydrate) [summary of product characteristics]. Available from: http://www.medicines.org.uk/emc/medicine/15211/XPIL/Migard. Accessed October 29, 2013.

29. Endo Pharmaceuticals. Frova (frovatriptan succinate tablets) [prescribing information]. Dublin: Endo Pharmaceuticals; 2013. Available from: http://www.accessdata.fda.gov/drugsatfda_docs/ label/2013/021006s019lbl.pdf. Accessed October 29, 2013.
Drug Design, Development and Therapy

\section{Publish your work in this journal}

Drug Design, Development and Therapy is an international, peerreviewed open-access journal that spans the spectrum of drug design and development through to clinical applications. Clinical outcomes, patient safety, and programs for the development and effective, safe, and sustained use of medicines are a feature of the journal, which

\section{Dovepress}

has also been accepted for indexing on PubMed Central. The manuscript management system is completely online and includes a very quick and fair peer-review system, which is all easy to use. Visit $\mathrm{http}: / /$ www.dovepress.com/testimonials.php to read real quotes from published authors. 